$\begin{array}{cl}\begin{array}{c}\text { Revue } \\ \text { de } / \text { histoire }\end{array} & \text { Revue de l'histoire des religions } \\ \text { des religions } & \begin{array}{l}4 \mid 2009 \\ \text { Varia }\end{array}\end{array}$

\title{
Une guerre sans épithète : les troubles des Cévennes au prisme catholique
}

Déchirures civiles et violences de religion (vers 1685 - vers 1710)

A War without Epithets: the Cevennes Troubles Seen through a Catholic Prism.

Civil Divisions and Religious Violence (ca. 1685 - ca. 1710)

\section{Chrystel Bernat}

\section{OpenEdition}

Journals

Édition électronique

URL : http://journals.openedition.org/rhr/7546

DOI : $10.4000 /$ rhr.7546

ISSN : 2105-2573

Éditeur

Armand Colin

Édition imprimée

Date de publication : 1 décembre 2009

Pagination : 639-650

ISBN : 978-2200-92592-5

ISSN : 0035-1423

Référence électronique

Chrystel Bernat, «Une guerre sans épithète : les troubles des Cévennes au prisme catholique », Revue de l'histoire des religions [En ligne], 4 | 2009, mis en ligne le 01 décembre 2012, consulté le 19 avril 2019. URL : http://journals.openedition.org/rhr/7546 ; DOI : 10.4000/rhr.7546 


\section{Une guerre sans épithète : les troubles des Cévennes au prisme catholique Déchirures civiles et violences de religion (vers 1685 - vers 1710)*}

En 1702 l'insurrection camisarde inaugure en Languedoc un temps de troubles religieux. La révolte mute en un conflit polymorphe. On examine ici la déclinaison des duels qui mirent aux prises instances politiques, clergé et populations, et l'entrée d'une société en guerre. L'approche souligne la multiplicité des violences - physiques, idéelles, langagières -, et des modalités d'affrontements confessionnels. A partir d'archives inédites, la thèse postule une reconsidération globale des troubles en incluant un troisième acteur proprement civil et en valorisant l'étude d'une guerre en son système. De ce défi protestant, unique dans l'histoire révocationnaire, elle interroge aussi l'état du catholicisme méridional arrivé au terme de son parjure.

\section{A War without Epithets : the Cevennes Troubles Seen through a Catholic Prism. Civil Divisions and Religious Violence (ca. 1685 - ca. 1710)}

The 1702 revolt of the Camisards following persecution is the start of a period of civil unrest in Languedoc. This dissertation analyses how a society enters into war: this revolt becomes a polymorphous conflict and opposing passions engage the political powers, the clergy and the civilian population. The types of religious confrontation and violence are multiple - physical, ideological, linguistic. This study offers a new overview of the conflict by drawing on previously unpublished archives and taking into account a third protagonist, purely civilian. It examines the war as a whole, beyond the confrontation between the Camisards and the political powers. Starting with this Protestant revolt, unparalleled in the "révocationnaire " period, it analyses the situation of Catholicism in Languedoc, which has at this point reached the limits of its self-betrayal.

* Thèse de doctorat d'Histoire (mention Religions et systèmes de pensée) sous la direction de M. Hubert Bost, École Pratique des Hautes Études, Paris (EPHE), soutenue le 20 décembre 2008 (3 tomes, 966 p.). - Membres du jury : Mme et MM. les Professeurs Hubert Bost (EPHE), Olivier Christin (Université de Lyon II), Arlette Farge (Centre National de la Recherche Scientifique), Philippe Joutard (Université de Provence), Didier Poton (Université de La Rochelle). - Mention très honorable avec les félicitations du jury à l'unanimité. 


\section{RÉSONANCES CIVILES DES TROUBLES DE 1702 : OBJET ET TERRAINS D'ENQUÊTE, POSTULAT ET PROBLÉMATIQUE}

Ces travaux s'inscrivent dans la lignée des premières études sur le sujet, qui déjà s'intéressaient à la violence catholique durant les troubles des Cévennes. Tandis que ces recherches initiales faisaient cas des affrontements les plus radicaux, en l'occurrence les plus subversifs, la thèse s'est résolument orientée sur l'attitude générale des populations catholiques confrontées à la quête libertaire des révoltés protestants. Ceci en partant d'un postulat simple : face à la prise d'armes camisarde qui devient insurrection durable, puis guerre à part entière, pouvait-on concevoir une guerre sans ennemis autres que la soldatesque et, a fortiori, appréhender la révolte camisarde comme une émotion close en sa propre raison, rivée à sa revendication exclusive de la liberté de conscience? En somme, fallait-il s'arrêter à la dualité que lui a prêtée jusqu'à nos jours l'historiographie, en opposant presque invariablement les Camisards aux troupes du roi et condamner définitivement l'histoire des troubles du Languedoc à une approche en miroir? Interprétation restrictive qui revenait à dénier aux mobilisations populaires toute autonomie et à dissoudre l'aspect séditieux d'une partie d'entre elles, mais aussi à niveler les dissonances intra-confessionnelles et à esquiver ce que le conflit contient de plus touffu, à savoir ses partitions combatives et sa violence plus strictement confessionnelle. Le parti pris fut alors d'étudier les résonances de l'insurrection à l'échelle sociétale et catholique, au-delà du seul regard du pouvoir, et d'interroger la part de catholicité du conflit en démêlant l'écheveau des implications civiles dans la guerre. Ce mot même de conflit dit l'objet des travaux qui visaient à étudier la révolte dans ses interdépendances. Ce à quoi nous invitait la dénomination approximative de cette prime révolte camisarde que les contemporains ont désignée tour à tour comme une rébellion, des soulèvements, une maudite guerre, des troubles, une révolution. Il revenait ainsi d'en considérer la confusion sémantique et l'effervescence, point nodal d'une interrogation historique qui chercha à comprendre, malgré la profusion des dénominations, l'absence de qualification de ces désordres. Guerre sans épithète - sinon définitive - dont le non-dit, autant que l'indécision 
des témoins à la considérer comme une révolte populaire, une insoumission religieuse ou une guerre de partis formés, n'était probablement pas sans signification. De là, la volonté de déterminer, en parallèle de la lutte avec les tenants de la répression officielle, ce que la prise d'armes camisarde a provoqué dans les milieux catholiques et de pénétrer, au-delà de la violence d'État, la violence civile et les modalités d'expression d'une société au combat. Telle est l'idée de la métaphore du «prisme catholique » : l'image d'un volume triangulaire prolongeant les intuitions des travaux précédents sur le « triptyque insurrectionnel » et l'« affrontement trilatéral » qui déjà réintroduisaient le tiers catholique.

Plusieurs interrogations connexes ont jalonné la recherche ouverte aux pratiques de violence à la fois autonomes, statutaires ou complices de la répression conjoncturelle. Tout en faisant cas des affrontements proprement indépendants - qui interrogeaient les points de basculement populaires dans la guerre -, quelle pouvait être la spécificité de l'usage d'une violence civile peu ou prou arrimée à la violence du pouvoir? La question posait celle du cadre spécifique des formes de clivages confessionnels sous le régime révocationnaire. Comment s'expriment-ils et où se manifestent-ils? La violence exige-t-elle étendard? Se déploie-t-elle en une seule geste agressive? D'ailleurs, le religieux explique-t-il toutes les marques d'affrontement confessionnel? En outre, qu'indiquent les engagements disparates des acteurs catholiques de l'état du catholicisme méridional? Traduisent-ils une vigueur ou une défaillance? Dans quelles mesures les troubles peuvent-ils apparaitre comme une mise à l'épreuve du catholicisme post-révocationnaire? Enfin que donne à voir, hors du théâtre, l'écho de la guerre en matière de coexistence religieuse?

Considérant la violence comme un langage, il revenait ainsi d'en dégager les ressorts et les dynamiques croisés. La volonté a été d'entrer dans les remous de la guerre et de restituer le concert de dualités saisies dans leurs antécédences et leurs résurgences - d'où l'ancrage chronologique dans les années 1680 et les échappées ponctuelles dans les années postérieures aux troubles -, tout en tentant de démêler la partition des militances, les logiques partisanes et l'entrelacs des antagonismes religieux qui se disent en une foule de pratiques hostiles venues déjouer les définitions de la guerre, liées d'ordinaire aux luttes armées et sanglantes. L'objectif appelait ainsi 
à interroger la multiplicité des engagements populaires ouverts à de nouvelles expressions de rivalités jusqu'alors minorées en regard de la coercition royale et des affrontements civils les plus revendiqués. Pour ce faire, l'enquête a porté sur la matière brute qui bénéficie, dans une équité rare, des sources de la plupart des protagonistes des deux confessions. C'est dans cette matrice archivistique qu'est apparue dans ses contradictions et sa radicalité, une guerre civile diluée dans une répression criarde. C'est cette guerre que l'analyse, dans le cadre d'une étude globale, a cherché à restituer jusque dans ses expressions marginales, ses intensités inégales et ses déboires au long cours.

\section{GESTE OPPOSITIONNELLE ET CHAMPS CROISÉS DE L'AFFRONTEMENT : MÉTHODES ET CHOIX ÉPISTÉMOLOGIQUES}

L'investigation a alors usé d'un va-et-vient constant entre ces sources primitives, l'épistémologie, les études et monographies historiques au profit d'une approche en focales variables - macro, micro et méta historiques - capable de restituer l'entrelacs des champs d'action et l'étagement des velléités d'opposition qui, à l'échelle du hameau, du village, du bourg, du diocèse, de la province, des cercles politiques et diplomatiques, pouvait répondre de la spécificité du sujet, traversé de composantes hétéroclites et constitué d'un enchevêtrement de théâtres d'engagement qui ne se limitent pas à celui principal du Bas-Languedoc. Ce parti pris méthodologique avait aussi l'avantage de saisir le conflit autant dans ses grandes tendances que dans ses soubassements et manifestations isolées ou insolites, en relayant convictions individuelles et motivations communautaires, pensées intimistes et anathèmes collectifs, engagements autonomes et mobilisations solidaires.

Du reste, l'étude ne visait pas à élaborer une chronique des affrontements. La volonté fut d'approcher le geste plus que le fait et de restituer un climat plus que de procéder à un relevé exhaustif de la violence. Ce qui n'excluait pas d'établir une trame factuelle, dense et représentative des temps et des formes d'antagonismes durant la guerre (1702-1710). D'où le choix de livrer en annexe la matière événementielle à valeur de pièce justificative servant analyses sérielles et statistiques, tout en s'affranchissant d'une histoire positiviste 
dans le corps du texte, réservé aux mouvements de fond, à la fois à l'étude des expressions de rivalités et des postures adversatives, et à l'analyse des mécanismes et des articulations de la violence confessionnelle dans un intervalle plus long (1670/1685-1710).

Cinq autres principes méthodologiques ont présidé à cette recherche. D'abord, celui de restituer la complexité des troubles sans chercher à fondre des dissemblances en un système cohérent mais, au contraire, d'en laisser affleurer les éléments discordants. Ensuite, a primé l'exigence de considérer progressions et mutations; ainsi, de ne pas tenir une posture pour définitive, un geste pour unilatéral, une signification pour immuable, une motivation pour exclusive, mais de faire cas des évolutions et des revirements, des nuances et des ruptures d'accord. Il fut aussi une priorité de saisir les violences dans leurs interactions et de ne pas couper l'étude des mouvements de celle des effets concrets, fussent-ils parfois indirects ou différés. De même, souhaitant éviter une étude strictement typologique, il demeura essentiel de décrypter mobiles, argumentaires, imaginaires et représentations présidant à la violence. À ce titre, l'analyse lexicale s'est avérée, avec celle des formes discursives, un outil de choix. Enfin, l'étude fixa pour objectif de s'interroger - tour à tour mais aussi de manière réflexive - à la fois sur l'individuel et le collectif, le singulier et le partagé, et sur ce que l'événement isolé et l'attitude personnelle supposent parfois de communautaire.

\section{ÉTUde DE LA VIOLENCE ET RÉÉVALUATION DU DUALISME CONFESSIONNEL AU DÉBUT DU XVIII ${ }^{\mathrm{E}}$ SIÈCLE}

Un premier mouvement de l'étude, consacré aux arcanes de la guerre - et à la notion de violence diffuse - s'est attaché à restituer une violence disséminée et tangentielle (chapitres 1 à 3 ). Cette violence insidieuse, délibérée ou sollicitée, autonome mais le plus souvent tapie dans les rouages coercitifs du pouvoir a révélé l'implication croisée du gentilhomme et du notable, du paysan et de l'artisan qui, en tant qu'acteurs, mandataires ou solliciteurs, exécutants, assistants et collaborateurs, entrent dans la guerre par une infinité de voies oppressives. L'enquête a ainsi propulsé sur la scène quantité d'adversaires que l'absence d'appartenance revendiquée aux groupes radicaux n'a pas toujours hissés au rang d'opposants. 
Constat qui a imposé de rompre avec l'idée d'une minorité activiste et d'une masse attentiste, au profit d'une société en guerre dans laquelle toutes les franges prennent part, le plus souvent à l'abri de l'écran répressif. Mais ce premier volet a surtout montré combien la violence ne nécessite pas irrémédiablement une idéologie mais profite d'une oppression légale, combien elle peut ne pas exiger des initiateurs mais parfois seulement des partisans qui, sans réclamer un champ propre d'action, usent d'une latitude et bénéficient d'une conjoncture. De même, l'opposition ne se limite pas au coup directement porté et n'est pas nécessairement armée ni sanglante, mais procède d'une multiplicité de marques, là où filtre une hostilité, s'exprime une animosité, s'énonce une rivalité, s'écrit une pensée agressive, s'ancre une geste belliqueuse, déclinaisons plurielles de l'engagement contre l'adversaire qui a eu pour effet de revisiter la géographie du clivage - distante du champ de bataille - et de redéfinir la figure de l'ennemi protestant, qui compte autant de facettes qu'il y a de figures d'opposants catholiques.

\section{Approche des radicalismes religieux : ZÈles, fanatismes ET DÉVIANCES SOUS LA MONARCHIE LOUIS-QUATORZIENNE}

La seconde articulation de l'étude est consacrée aux oppositions catholiques rassemblant les postures civiles les plus radicales (chapitres 4 et 5). L'engagement affiché et durable de ces ultras rompt avec l'activisme feutré de la masse, au profit d'une hostilité revendiquée et d'une violence exacerbée plus nettement indépendante et davantage collective. Là aussi la diversité des engagements est de mise. Groupuscules séditieux nécessitant sanctions punitives et formations militantes supplétives multiplient les implications extrémistes où s'enchevêtrent déchirures civiles, spectre de guerre de religion et symptômes de guerre paysanne. L'étude détaillée de ces mouvances belliqueuses a mis en exergue de fortes disjonctions intra-confessionnelles. Elle a révélé aussi des manifestations d'animosité populaires qui, en apparence désinvoltes et aveugles ou dévoyées et désordonnées, sont apparues - notamment chez les Florentins - comme le versant tumultueux d'un militantisme fort; l'aspect subversif de l'engagement, autrement dit la forme, ayant peu à voir avec la détermination et l'acharnement à affecter l'adver- 
saire confessionnel. De même, la faiblesse des attaques frontales ne signifie pas ici l'inexistence d'un combat, ni l'absence de nuisance. Pas plus que l'affrontement armé ne constitue la marque absolue d'une lutte qui se manifeste encore dans la défense d'un territoire et des mouvements d'entrave. Dans cette guerre déclarée aux huguenots, il ne semble pas qu'il y ait contradiction entre l'agression sauvage des religionnaires et le combat proprement anti-camisard. Vindicative ou arbitraire, la violence catholique décline une guerre ouverte contre l'ennemi protestant. Force a été aussi de constater que le banditisme le plus éhonté - manifeste chez les Cadets de la Croix - n'est pas sans recouvrir la manifestation d'un antagonisme confessionnel marqué. Le désordre recèle aussi le combat, fût-il altéré, corrompu ou déréglé de l'avis des puissances. L'étude montre que le désintérêt apparent pour la lutte n'est pas sans déploiement de haine, que le factieux ne signifie pas - en regard du choix sélectif des victimes - le non militant, et que la violence même la plus triviale n'est pas sans être profondément partisane. Une guerre confessionnelle se joue ainsi à couvert de l'insoumission et de la rébellion camisardes dans ces mouvements croisés de contre-révolte et de fureurs catholiques venus s'introduire sinon s'interposer dans le face-à-face entre Camisards et autorités répressives. Guerre qui ne se limite donc pas à la violence de la réplique du pouvoir mais qui procède de cette conjonction d'initiatives civiles redoublant préjudices et tensions. Une guerre religieuse que viennent encore renforcer l'analyse approfondie de l'attitude hautement belliciste du clergé bas-languedocien et l'interposition coercitive de l'enclave pontificale d'Avignon, renforcée par la crainte tenace d'un assaut hérétique dans les terres de la papauté (chapitre 6).

\section{Des troubles PRotestants À LA GUerRe CIVILE}

L'attention accordée aux clivages passés a aussi imposé de ne pas ancrer les mobiles d'action catholiques dans une dynamique exclusivement réactive. L'offensive au contraire y est prépondérante, l'agression délibérée et l'occasion conjoncturelle de manifester son altérité régulière. Ainsi, les velléités libertaires des protestants ont provoqué ou réactivé des alliances répressives avec le pouvoir, mobilisé des fronts de refus, dévoilé des dispositions 
hargneuses, révélé ou animé des haines enfouies. Partant, devaiton alors tenir pour définitif que l'histoire des troubles fut exclusivement celle de la révolte camisarde, ou considérer que la prise d'armes initiale des insurgés protestants introduisait un plus large conflit? L'aspect polymorphe des engagements catholiques - qui épousent la collaboration avec les instances persécutrices, optent pour la contre-révolte indépendante ou l'auxiliariat répressif -, la diversité des espaces mobilisés et l'ampleur de l'empreinte spatiale des oppositions, mais aussi la foule d'acteurs et la diversité de leurs profils dont les velléités d'action transcendent statut et profession, de même que la variété des cadres d'implication - individuel, familial, corporatiste, confessionnel ou communautaire - ont donné aux troubles une dimension plus vaste de guerre dans laquelle s'immiscent ou s'imposent pléthore de protagonistes entrés par diverses voies officielles et officieuses. Quels que soient les mobiles - hostilité confessionnelle, rancœur sociale, désaveu de la politique coercitive, volonté d'autodéfense, solidarité confessionnelle, profit des désordres, militantisme contre-camisard ou anti-protestant, peurs, ferveur religieuse ou défense de la foi -, quels que soient l'intensité et les rythmes de mobilisation, l'entrée échelonnée mais continue des populations catholiques dans les troubles, a ajouté à la dimension de conflit. L'implication massive comme la multiplicité des violences, des cibles et des victimes religionnaires - regroupant particuliers et communautés - ont donné à la guerre des Cévennes ses tonalités de guerre civile, fût-elle maintenue dans le cadre d'une répression royale d'abord orchestrée par les autorités locales. À ce titre, il apparaît que la guerre des Cévennes n'est pas seulement un acte d'émancipation spirituelle affronté à un appareil répressif d'État, mais un conflit religieux traversé et animé par une constellation d'oppositions civiles.

\section{LE CATHOLICISME LANGUEDOCIEN DANS LA TOURMENTE}

Face à la multitude des facettes du catholicisme au combat filtrait indéniablement une certaine vitalité. Pourtant, devait-on parler d'un catholicisme, d'un camp catholique? La multiplicité des engagements n'est pas sans illustrer le morcellement des forces et la disparité des visées combatives. C'est là le premier bémol à une 
entité catholique fragmentée, vigoureuse et pourtant défaillante qui invitait à interroger l'impact de la détermination camisarde. Car les mobiles mêmes qui poussent les catholiques à agir disent tous, sans exception, l'épreuve engagée. L'ardeur est à hauteur du défi, la violence militante à proportion du séisme camisard. C'est là l'objet de la dernière articulation analytique qui visait, à l'aune des représentations catholiques et des dérèglements qu'introduit, dans les consciences et sur le terrain, la révolte protestante, à restituer les indices d'une tourmente (chapitres 7 à 9). L'ultime volet de l'étude s'est ainsi attaché, à revers d'une pensée victimaire renouant avec le martyrologe, mais aussi en contrepoint d'une vitalité protestante et d'une violence camisarde visant la désolation du papiste, à déceler les champs d'une épreuve catholique : épreuve conjointe de la désolation, de la désorganisation, des dissensions et de la désunion, de la violence et de l'insoumission protestantes, épreuve de la chimère révocationnaire, renchérie par un désordre de militances civiles qui ajoutent au témoignage d'une religion bouleversée et ébranlée. C'est en effet la première fois depuis 1685 que l'étendard de la résistance calviniste porte haut la conviction intacte des cœurs et sanctionne violemment l'incomplétude du programme de conversion imposé par la contrainte.

De cette période de ferveurs antagonistes, les réflexions conclusives soulignent ainsi le temps suspendu de plusieurs faceà-face venus bousculer la cohésion présumée des camps. Troubles et disharmonie du catholicisme méridional, éprouvé dans ses certitudes, gêné dans ses contradictions, désordonné dans ses répliques donnent alors à voir, sous le régime louis-quatorzien, le temps inédit d'un vacillement. Celui d'un catholicisme acculé au spectacle de sa défaite, avant tout pastorale, et de sa déroute, infligée tambour battant. Ce n'est pas seulement le temps d'une désillusion - précoce -, ni le témoignage de l'impasse de la conversion forcée - tôt manifeste -, mais celui, éclatant, de son envergure. La guerre offre ainsi le visage d'un catholicisme post-révocationnaire acculé à son mépris du parjure et à l'extrémisme de sa persécution. Si le combat ne se fait pas sans redoublement de châtiments, la répression est à hauteur de l'impasse, et sur le terrain s'impose, inédit pour le clergé catholique en cette période de domination écrasante, le temps d'un repli insolite face à une révolte camisarde qui porte aussi saccage du catholicisme. Indéniablement, ces troubles ne sont pas le témoi- 
gnage exclusif d'une fébrilité catholique. Pourtant, là aussi, l'incohérence et la dispersion des ripostes en signalent les limites. Limites renforcées par l'arbitrage politique qui entend garder la haute main sur l'affaire des fanatiques des Sevennes et gère dans la contradiction les velléités de combat de ses sujets catholiques.

\section{RECONFIGURATION D'UN CONFLIT ET NOUVEAUX PARADIGMES du Clivage religieuX aU tournant du Grand Siècle}

Plus largement, l'étude des résonances de l'insurrection camisarde dans les milieux catholiques postule une reconsidération globale des troubles du Languedoc. Guerre qui ne saurait se borner à l'histoire dualiste opposant insurgés et troupes royales, mais s'ouvre à un troisième grand acteur proprement civil qui amène à privilégier le schème interprétatif d'un triptyque insurrectionnel. À l'issue d'une analyse comparative avec les guerres de Religion qui fait cas de nombreuses analogies, il apparaît clairement que la guerre des Cévennes s'apparente à une guerre de partisans portée par des violences de religion plus qu'à une guerre de Religion à proprement parler, ne serait-ce que par son contexte et ses revendications qui ne permettent pas de la considérer comme une simple réduplication des troubles du $\mathrm{XvI}^{\mathrm{e}}$ siècle. Pourtant, en extrayant la révolte camisarde de son écrin principal, l'étau oppressif, relevé à l'échelle d'autres provinces comptant de fortes minorités protestantes - notamment en Saintonge, en Guyenne, en Poitou - a projeté l'insoumission protestante en une autre dimension qui, d'événement circonscrit, s'avère relever d'une affaire commune suscitant à distance peurs, crispations, mobilisations préventives et velléités populaires d'agression. Ainsi, en parallèle des résonances au sein de l'Europe protestante, l'écho se trouve simultanément, sinon d'abord, à l'intérieur du royaume parmi les populations catholiques. Si ces interférences ont pointé des interfaces répressives catholiques - parmi lesquelles figurent les représentants de la curie romaine -, elles ont aussi débusqué des manifestations de clivages religieux latents en d'autres contrées que l'écho, même distant, des troubles camisards suffit à éveiller parmi les catholiques au profit d'un imaginaire collectif toujours vif et sombre contre le huguenot. 
Enfin, la guerre sans épithète que lèguent les troubles du Languedoc - guerre dense et composite qui ne se donne pas d'emblée pour telle et ne se qualifie pas -, ne postule pas un modèle insurrectionnel déclinable, là où, indiscutablement, l'intensité des déchirements religieux est sans exemple dans l'histoire postrévocationnaire du royaume. Pourtant, cette guerre sans nom rend compte d'un phénomène sourd qui peut-être traverse, à rebours des situations de coexistence pacifiques, les tensions entre catholiques et protestants de France. Sans doute porte-t-elle jusqu'à leur paroxysme plusieurs modes communs d'affrontements confessionnels que couvre et favorise la persécution des temps, mais que circonscrit et borne l'arbitrage royal adossé à un système étatique fort qui ne laisse pas - lorsqu'il en serait question - de prise à la guerre de Religion. Sous ce règne du moins, l'écran royal est là pour désagréger les velléités dualistes. C'est en partie pour cela que cette guerre n'a pas de nom, et ne saurait en avoir un, même si elle a existé. La puissante machine absolutiste ne la permet pas, la violence endossée par le pouvoir la dissipe et ne la compte pas, sinon pour supplétive. Mais cette guerre que l'on ne laisse pas se dire demeure aussi sans épithète pour la raison simple qu'elle n'a pas nécessité absolue de se définir comme telle. L'étendard de l'engagement proprement confessionnel n'est pas nécessairement revendiqué par les acteurs. En dehors des franges radicales, la plupart des violences catholiques civiles se déploient sans porte-drapeau. Quel en serait le besoin en regard d'une oppression légitimée par le pouvoir? D'où cette pléthore de coups bas portés sans oriflamme qu'il resterait à évaluer en d'autres terroirs du royaume comptant semblables frontières religieuses et similaires isolats confessionnels.

Ainsi, le terme de guerre sans épithète voudrait rendre compte de ce cadre singulier des rapports de force confessionnels biaisés par la persécution royale. Cadre informel où peut se manifester et se poursuivre un clivage religieux privé - ou dégagé - des emblèmes de lutte, et s'agencer un espace de violences légales coulées dans l'oppression ordinaire. Un constat qui appelle désormais à interroger, à plus vaste échelle, l'empreinte diffuse des modalités de clivages confessionnels sous le régime révocationnaire. 
